\title{
ETALONNAGE DES LASERS DE POURSUITE
}

\author{
José Pires
}

TRESCAL Parc d'Affaires SILIC BP 30441, 8rue de l'Estérel, 94593 Rungis, France

\begin{abstract}
.
L'étalonnage des lasers de poursuite, appelés plus communément laser tracker (LT), fait l'objet de plusieurs normes répondant à l'évolution des exigences et des technologies mais dont la mise en œuvre peut s'avérer complexe et pour lequel le nécessaire raccordement au Système International d'unités (SI) constitue également une problématique.
\end{abstract}

D'autre part, les principaux constructeurs ont établi leurs propres protocoles de vérification avec pour certains, la mise en œuvre d'outils spécialisés utilisés dans certain cas pour l'ajustage des composants technologiques, tels que codeurs et laser intégrés dans le LT.

Par ailleurs, la mise en place de solutions techniques pour l'étalonnage des LT s'inscrit dans un contexte industriel impliquant des contraintes de disponibilité et de coût.

Fort de ce constat, Trescal a étudié et mis en ouvre une approche industrielle et des solutions métrologiques répondant à ces contraintes afin de garantir que les LT confiés par ses clients conservent des caractéristiques métrologiques conformes aux spécifications techniques définies par le constructeur, en garantissant le raccordement des mesures au (SI).

La solution développée par Trescal s'appuie sur un laboratoire dimensionnel de haut niveau, une accréditation COFRAC de mesure de grandes longueurs intégrant un banc de $21 \mathrm{~m}$ couplé à un interféromètre laser. Elle intègre différentes options et possibilités en fonction des caractéristiques du moyen et de l'expression des besoins de l'utilisateur.

L'objectif de cette conférence est de présenter les solutions développées avec le support des constructeurs et du département mécanique de l'INSA de Lyon, et de présenter le retour d'expérience après plusieurs mois d'exploitation.

\section{CALIBRATION OF LASER TRACKERS}

Calibration of laser tracker (LT) is subject to several standards in order to meet changing requirements and technologies but the implementation can be complex and the necessary traceability to the International System of Units (SI) is also a problem.

More ever, major manufacturers have developed their own protocols for verification with, for some of them, the use of specific tools for the adjustment of technological components, such as laser and encoders implemented into the LT.

On the other hand, implementation of technical solutions for the calibration of LT has to take into consideration an industrial context involving availability and cost constraints.

With this in mind, Trescal designed metrological solutions, with an industrial approach to face all these issues, in order to guarantee the traceability of the measurements to the (SI) and the compliance of the LT calibrated with the technical specifications defined by the manufacturers.

The solution developed by Trescal relies on a high level dimensional laboratory, a COFRAC accreditation for long length measurements incorporating a 21 meters long bench coupled to a laser interferometer. It integrates various options and possibilities depending on the characteristics of the equipment and the user's needs. 
Web of Conferences

The objective of this conference is to present the solutions developed with the support of manufacturers and INSA Lyon mechanical department, as well as to present the feedback after several months of operation. 


\section{Contents}

Contents 3

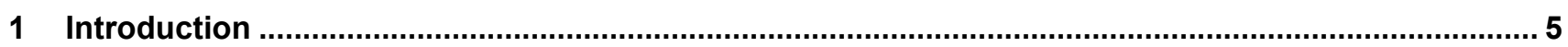

2 Laser tracker presentation - The different technologies ............................................................ 6

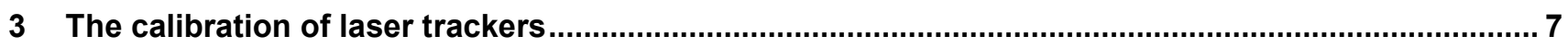

3.1 The recommendations in standards ASME B89.4.19 and ISO 10360-10 .................................... 7

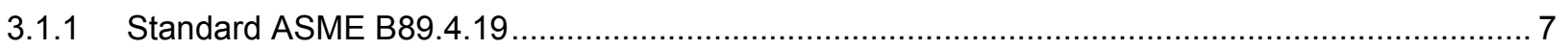

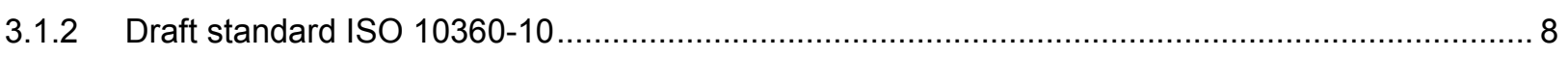

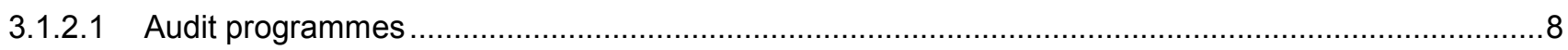

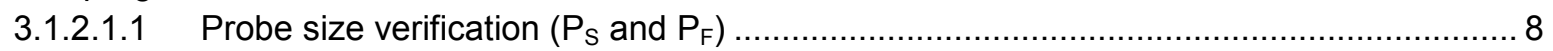

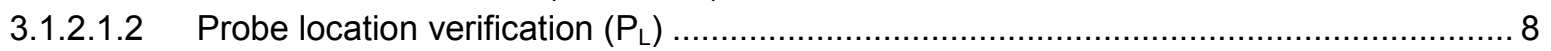

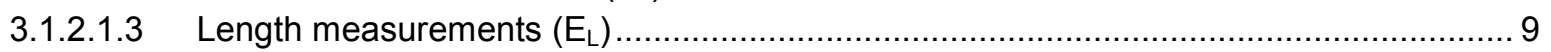

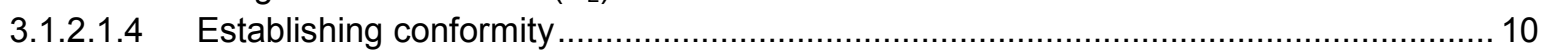

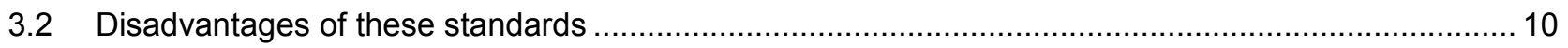

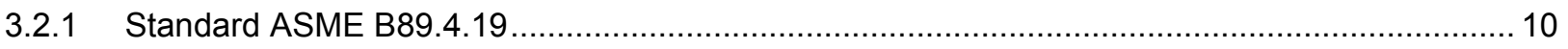

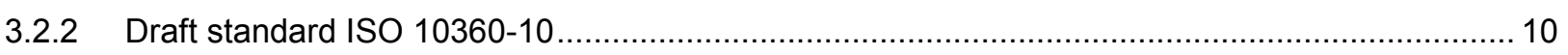

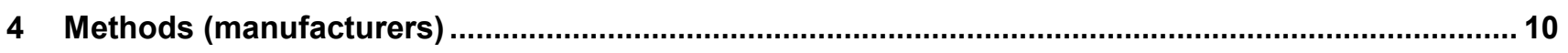

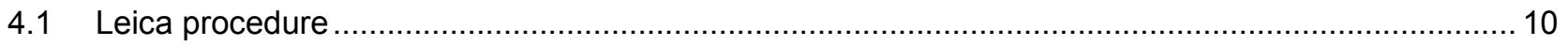

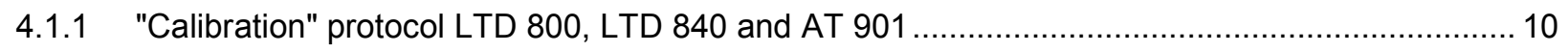

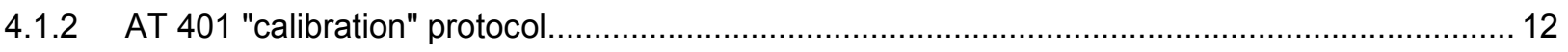

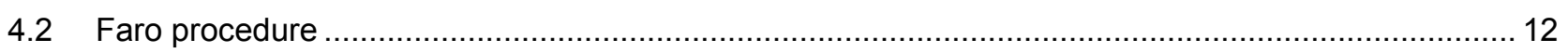

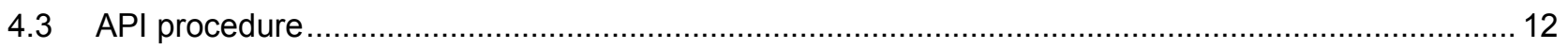

4.4 Disadvantages of the (manufacturers') methods ..................................................................... 13

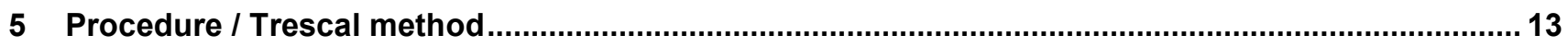

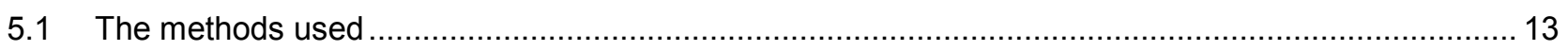

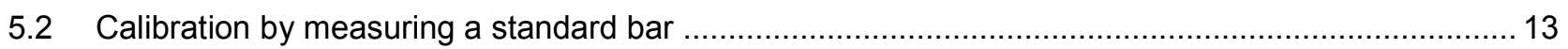

5.3 Calibration by comparison with a laser interferometer ............................................................. 13

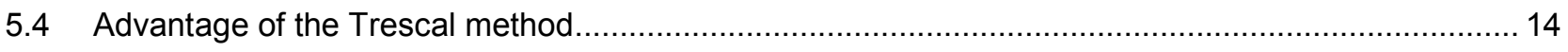

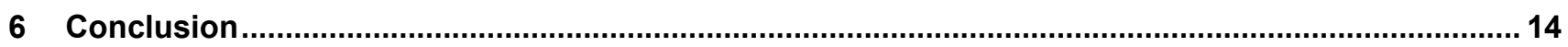




\section{Learning Objectives}

$\underline{\text { Knowledge }}$

This document identifies the different laser tracker technologies, their use and existing metrological verification protocols.

Understanding:

This document describes the recommendations in standards and the instructions established by manufacturers and presents the impacts for metrological verification.

Application:

This paper demonstrates how Trescal has implemented an industrial approach and offers metrological solutions by guaranteeing the traceability of measurements to the SI when calibrating laser trackers in an industrial context, including availability and cost constraints.

Analysis:

This document explains the operation of laser trackers, analyses the standardisation of calibrations, and identifies solutions on the market and the proposal from Trescal.

Summary:

With the growing use of laser trackers in industrial sectors such as aeronautics and automotive, by using this document, users will be able to choose the best calibration solution for their equipment.

Evaluation:

Through this document, the reader will be able to assess the advantages of the laser tracker calibration method, recommended by Trescal, with regard to requirements in standards and industrial constraints. 


\section{Introduction}

Trescal, an international company specializing in calibration, is represented in 16 countries and provides a broad range of services for the test and measuring equipment market.

Trescal operates 62 calibration laboratories and employs over 1,500 people worldwide. It serves 25,000 customers in various sectors such as Aerospace, Aeronautics, Automotive, Chemical, Communication, Defence, Electronics Manufacturing, Medical as well as Power Generation \& Utilities.

Supplementing standard technical services (calibration, verification and repair of test and measurement instruments), Trescal also offers its expertise for the implementation and acquisition of measurement systems, customised equipment asset management software solutions, metrology as well as technical support and training.

Trescal also studies the requirements and implementation of methods for the calibration of new equipment on the market.

One of these business studies includes the utilisation of laser trackers:

- Control and calibration

- Measuring industrial products (e.g., inspecting aircraft wings)

In January 2012, Trescal acquired the metrology contract at a leading aircraft manufacturer's facility. The work was previously managed by the manufacturer's personnel. Consequently, Trescal invested heavily in its metrology capability including the purchase of six laser trackers, computers and associated equipment. We also recruited ten operators; the majority were new to metrology. New operating instructions and procedures also had to be created.

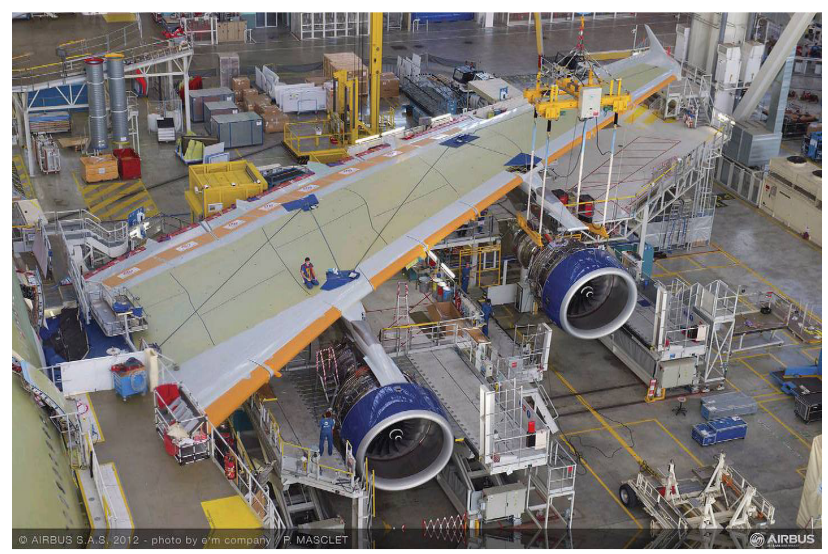

From the experience we gained training new operators we would estimate that it takes up to twelve (12) months to become fully-trained in all aspects of the work required. Trescal now provides a comprehensive package of work delivering measurements on time to programme.

For thousands of years, man has pursued the accurate measurement of volume using a coordinate system and geometry. First with the cubit, plumbs and sextants and later, in the 20th century, with coordinate measuring machines (CMMs) entering the stage. CMMs were able to accurately determine position in $\mathrm{x}, \mathrm{y}$ and $\mathrm{z}$ space and calculate volumes relatively quickly. However, as parts grew larger $(>1 \mathrm{~m} 3)$ in volume, CMMs had to move from the fixed-table to the portable type, like Faro arms, theodolites and photogrammetry, to accommodate the growth. However, with aerospace, automotive and defence applications, these methods quickly reached their limitations in the 1980s. In the late 1980s, laser trackers were developed. There efficiency comes in the reduction of personnel to make the measurement, the speed by which measurements are made as well as the distances to which components can be measured. During the late 1990's laser tracking technology and measurement accuracy escalated.

Laser trackers are rather simple in construction at a macroscopic scale; it consists of a laser source, typically $\mathrm{HeNe}$ gas, and a retroreflector. A retroreflector is a device that reflects light parallel to the incident ray but with little diffusion or scattering. A simple and common retroreflector is what a person may find on the reflector on a bicycle, a road sign or the reflective decorations on running shoes. In spite of the incident angle to the object the light emanating from the observer will return to the observer. This is different than a mirror in that the incident ray has to be normal (or perpendicular) to the plane of the mirror in order for the light generating position to see the reflected light beam. Most common laser tracking systems use a spherically mounted retroreflector (SMR) which splits the incident ray and returns a portion of it back to the laser tracker and another portion of the ray to the interferometer which measures the distance from the tracker to the SMR. To measure the angles from the tracker to the SMR, the tracker is equipped with two encoders, an azimuthal (X-Y plane) and a zenith (elevation). Coupled with the distance measurement using the interferometer and the angle encoders of the tracker, the accurate position of the SMR, or more importantly the part, can be calculated, or reckoned for those of you who were in the Navy.

\section{Laser Trackers Used:}

\section{$\underline{901 \text { Tracker }}$}

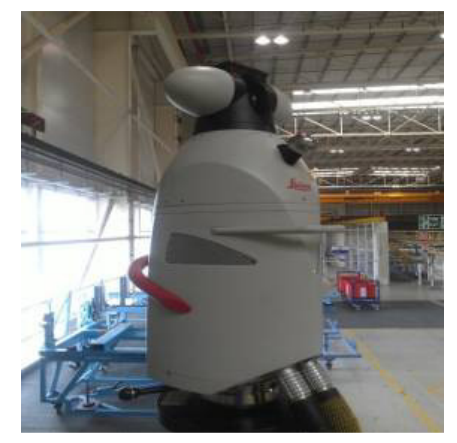

This model is used for all inspections that require a large quantity of points to be measured expeditiously, (e.g. Leading edge of wing and Root end of wing). It is considerably larger and less mobile than the 401. This makes it very sturdy though and is much more reliable 
over the length of a measurement. Unlike the 401, this tracker is self-levelling so time is saved during set-up.

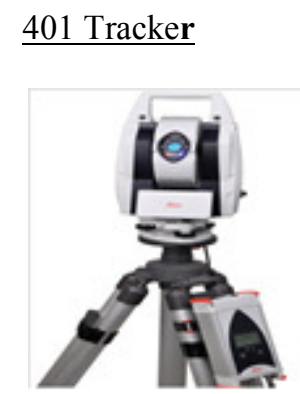

This is used for the quicker measurements as well as for its ease of set-up and use. It is lighter and much more versatile for taking measurements in awkward positions. This tracker enables staff to carry out measurements in almost any condition and in any position. It also can also operate using batteries so, when line power is an issue, this capability is very beneficial.

Field checks are carried out on the tracker before the initiation of every job and, in the case where a tracker needs to be moved to a number of positions for the same job, a verification test is performed every time a tracker is moved to the new position.

\section{Laser tracker presentation - The different technologies}

A laser tracker is a 3D measuring device for long distances, used mainly in the aeronautical and automotive fields and more generally in large-dimension engineering industries.

This device's measuring principle consists of emitting a laser beam, whose signal is sent by means of a cube corner target/reflector to the laser tracker. This reflector is used as a sensor for acquiring measurement data. To obtain the measurement, two types of metrology technologies are used, combined or otherwise:

- IFM (Interferometric Measurement)

- $\mathrm{ADM}$ (Absolute Distance Measurement).

The notable differences between these technologies are due to their operating modes, the length of the light carrier waves used and their accuracy.

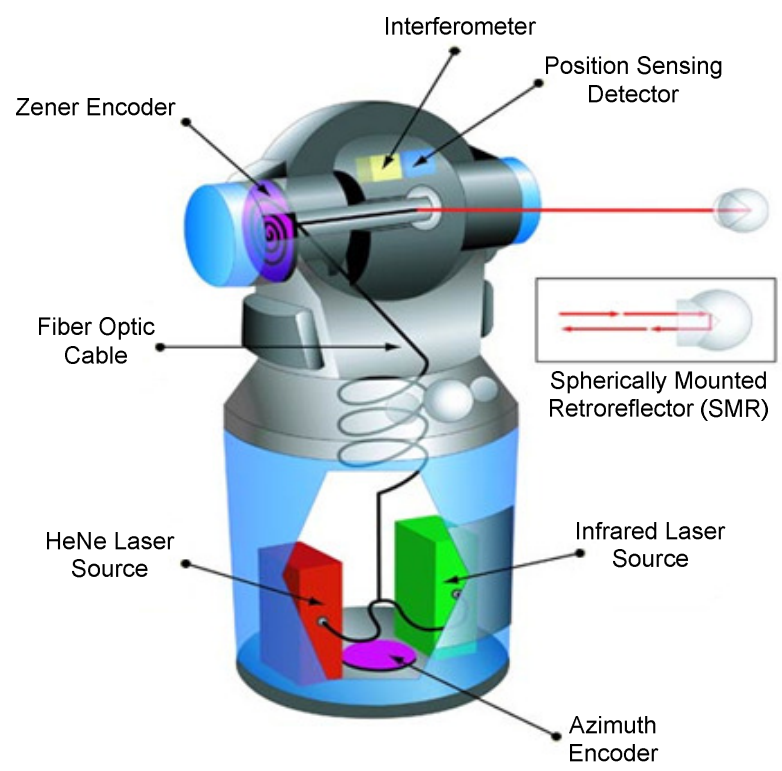

Figure 1. Schematic representation of how a laser tracker works

IFM systems break down a red beam laser source into a reference and a measuring component. The reference beam is retained in the laser tracker and the measuring beam is sent outwards, reflected on the target and then sent back to the laser tracker. When the measuring beam returns to the laser tracker it combines with the reference beam, thus allowing the creation of optical interference fringes. It is this interference pattern that allows the radial measurement. The interferometer waits until the return beam is powerful and undistorted in its path to and from the target, so that the interference fringe can be accurately created. This technology is very accurate but the disadvantage is that it provides only a relative distance (depends on a point of origin and the position of the centre of the reflector at a fixed point called the "nest").

ADM systems modulate in amplitude by using an oscillator and a carrier wave usually in the near infra-red wavelength region of the light spectrum. The distance is then deduced by the phase difference between the beams transmitted and reflected (returned). This technology is less precise than IFM but its advantage is that it supplies an absolute distance. For stations equipped with both technologies, ADM is used to re-initialise the interferometer when its laser beam is cut or lost.

The head of the laser tracker is equipped with two pivots which allow it to measure according to the azimuth angle (XY or horizontal plane) at $\pm 270^{\circ}$ according to the zenith (vertical) angle $+75^{\circ}$ up to $-50^{\circ}$ (see Figure 2 below). Transverse measurements are made using angular encoders situated in each of these pivot links. 


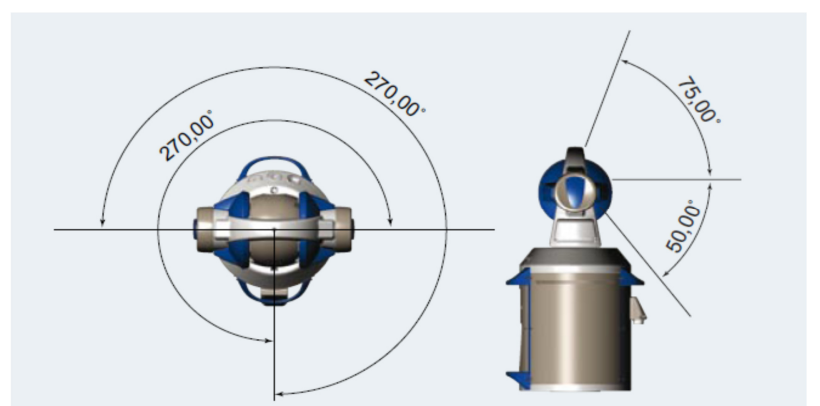

Figure 2. Diagram of the rotation of the laser tracker's measuring head (Faro-Ion)

According to the manufacturers, the maximum measuring distance of a laser tracker varies between 40 and $80 \mathrm{~m}$.

Targets/reflectors/SMR (spherically mounted retroreflector) standards are spheres comprising precision optics, precious metals, ultra high performance adhesives and more or less perfect geometry. The first objective is to reflect the laser beams with the greatest intensity without altering them when reflecting and/or refracting them on the different geometrical surfaces of the target. All manufacturers use the same target diameters which are 0.5 inches and 1.5 inches. Sometimes manufacturers offer a reflector with an intermediate diameter of 0.875 inches.

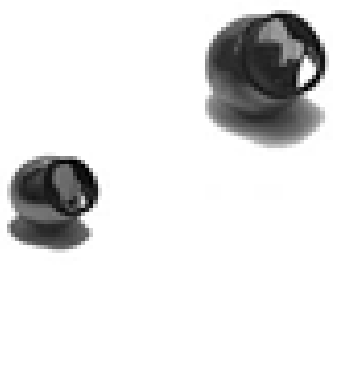
Figure 3. From left to right, reflectors/targets/
SMRs of $0.5,0.875$ and 1.5 inches.

The measurement a laser tracker depends on several parameters which constitute its error model. This is mainly due to the fact that laser trackers are made up of a set of sub-assemblies which have to be correctly aligned and oriented so the instrument provides correct measurement results. Most analytical models assume the distance and the horizontal and vertical angles are measured from the same fixed point located somewhere inside the device. In addition, the three main axes (trunnions- $X$, collimation- $Y$ and vertical- $Z$ ) defining the instrument's coordinates system must be orthogonal to each other and meet at that point.

Finally, every laser tracker has a controller. This controller connects the laser tracker to the computer. The computer may incorporate a weather station or receive an external signal from a temperature sensor to compensate for environmental conditions that may affect laser accuracy.

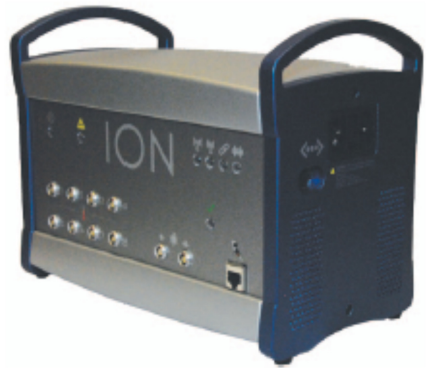

Figure 4. Illustration of a laser tracker controller (Faro Ion)

\section{The calibration of laser trackers}

\subsection{The recommendations in standards ASME B89.4.19 and ISO 10360-10}

Two standards deal, in part, with the calibration of laser trackers. The first one, ASME B89.4.19 is an American standard based on evaluating the performance and geometrical misalignment of laser trackers while the second, currently in draft, is an ISO standard centred on laser tracker verification and acceptance programmes for measuring point-to-point distances.

\subsubsection{Standard ASME B89.4.19}

This standard describes how to evaluate laser tracker performance by following the three programmes suggested below:

"Range test":

- Verification of distance by going through the encoders and testing only the distance meter. To do this, the reflector/target/SMR has to be placed at the height of the laser tracker head and, without changing the position of the horizontal encoders, the distance is measured while moving the target away from the laser tracker's location at specific aligned points.

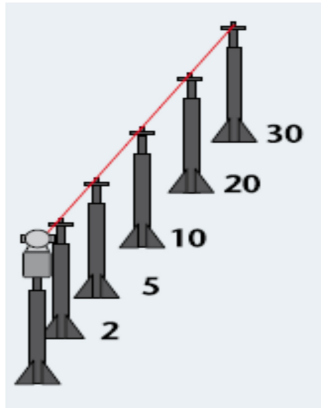

"Volumetric system test":

- Verification of volume using a bar of a calibrated length with reflectors/targets/SMRs on opposite ends. This bar may be arranged in any position in space (horizontal, vertical, diagonal, etc.); the volume is then geometrically calculated. 


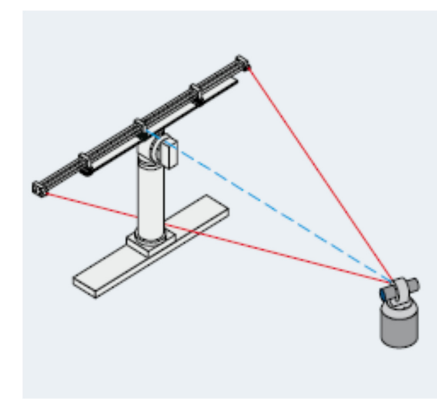

"Two-face test":

- This protocol verifies the horizontal collimation error. This verification is also done using the standard bar measured using the double centering method (DCM). This method consists of measuring a point on face 1 (normal measurement) then measuring it on face 2 , which is equivalent to measuring it by varying the angular encoder (azimuth) by $180^{\circ}$ (critical measurement). Employing this methodology can eliminate $90 \%$ of instrument and operator errors.

\subsubsection{Draft standard ISO 10360-10}

Performance is based on laser tracker verification and acceptance programmes. This document also considers three programmes but it focuses on the probing errors $\left(\boldsymbol{P}_{\boldsymbol{S}}\right.$ and $\left.\boldsymbol{P}_{\boldsymbol{F}}\right)$, probe location $\left(\boldsymbol{P}_{\boldsymbol{L}}\right)$ and length measurements $\left(\boldsymbol{E}_{\boldsymbol{L}}\right)$ to determine the tracker's performance.

The important terms within this standard are:

\begin{tabular}{|c|l|}
\hline Symbol & \multicolumn{1}{c|}{ Description } \\
\hline $\boldsymbol{P}_{\boldsymbol{S}}$ & Probing size error \\
\hline $\boldsymbol{P}_{\boldsymbol{F}}$ & Probing form error \\
\hline $\boldsymbol{P}_{\boldsymbol{L}}$ & Probing location error (via double switching) \\
\hline $\boldsymbol{E}_{\boldsymbol{L}}$ & $\begin{array}{l}\text { Length measurement error (Distance between } \\
\text { two points) }\end{array}$ \\
\hline $\boldsymbol{P}_{\boldsymbol{S}, \boldsymbol{M P E}}$ & Maximum permissible probing size error \\
\hline $\boldsymbol{P}_{\boldsymbol{F}, \boldsymbol{M P E}}$ & Maximum permissible probing form error \\
\hline $\boldsymbol{P}_{\boldsymbol{L}, \boldsymbol{M P E}}$ & Maximum permissible probing location error \\
\hline $\boldsymbol{E}_{\boldsymbol{L}, \boldsymbol{M P E}}$ & $\begin{array}{l}\text { Maximum permissible length measurement } \\
\text { error }\end{array}$ \\
\hline
\end{tabular}

Table 1. Key variables in the standard

\subsubsection{Audit programmes}

\subsection{Probe size verification $\left(P_{S}\right.$ and $\left.P_{F}\right)$}

Verifying the size of the probe (reflector/target/SMR) consists of measuring a standard sphere with a diameter of between 25 and $51 \mathrm{~mm}$. 25 points should be measured, distributed over a hemisphere.

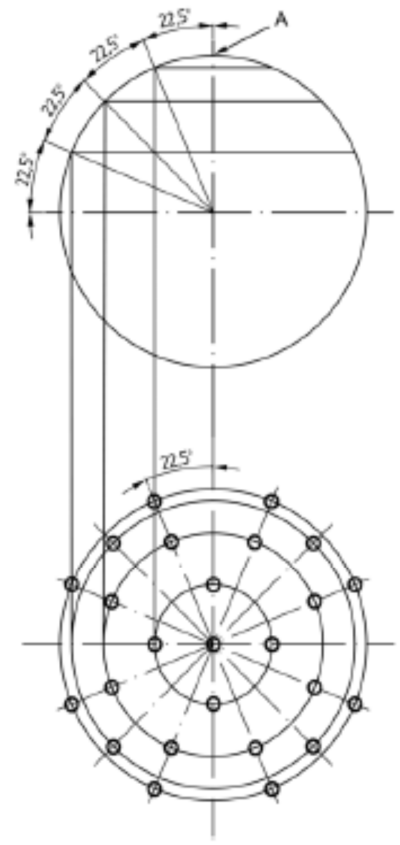

Figure 5. Location of the points to probe

These 25 points should be measured at two distances from the laser tracker. A first series of measurements at a distance of less than $2 \mathrm{~m}$ and the same height as the tracker head and a second series of 25 measurements at a distance of approximately $10 \mathrm{~m}$ at a height of $\pm 1 \mathrm{~m}$ from the height of the tracker head.

When these series of measurements are made, by approximation of the Gaussian law, the diameter of the probed sphere, $D_{\text {meas }}$, should then be determined and then the probe size error is calculated by subtracting the diameter obtained from the reference diameter of the standard sphere:

$$
P_{S}=D_{\text {meas }}-D_{\text {ref }}
$$

To determine the probe form error, the minimum radius of the 25 points probed is subtracted from the maximum radius:

$$
P_{F}=R_{\max }-R_{\min }
$$

\subsection{Probe location verification $\left(P_{L}\right)$}

Verification of the location of the sensor by the laser tracker consists of measuring, using the "double centering" method, three points situated at respective heights relative to the height of the tracker head:

- same height

- $1 \mathrm{~m}$ below

- $1 \mathrm{~m}$ above

Six series of measurements (below) should be taken for each of the three heights (18 measurements in all):

$\begin{array}{cc}\text { Laser Tracker Distance (m) } & \text { Azimuth Angle }\left(^{\circ}\right) \\ 1.5 & 0 \\ 1.5 & 120 \\ 1.5 & 240 \\ 6.0 & 0 \\ 6.0 & 120 \\ 6.0 & 240\end{array}$

Table 2. Points for probe location verification 


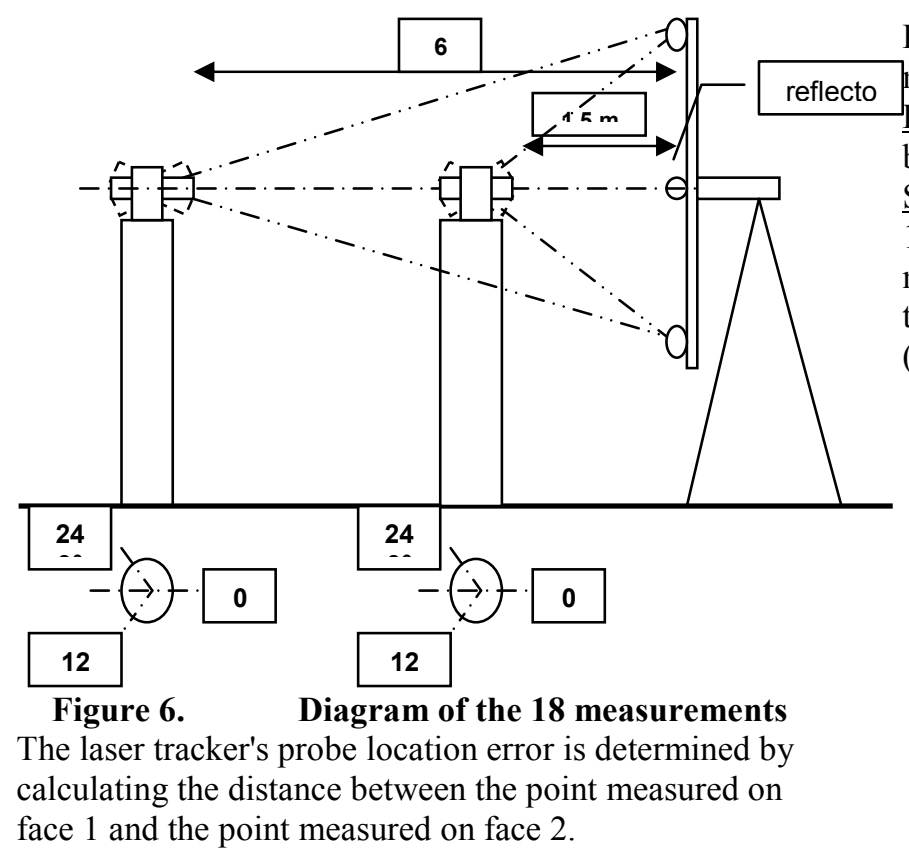

$$
\begin{gathered}
P_{L}=\sqrt{\left(x_{1}-x_{2}\right)^{2}+\left(y_{1}-y_{2}\right)^{2}+\left(z_{1}-z_{2}\right)^{2}} \\
\text { Point on face } 1\left(x_{1}, y_{1}, z_{1}\right) \\
\text { Point on face } 2\left(x_{2}, y_{2}, z_{2}\right)
\end{gathered}
$$

The location error accepted is the greatest error obtained over the 18 measurements.

\subsection{Length measurements $\left(E_{L}\right)$}

Length measurements consist of measuring the distance between two points in space. The distance between these two points must be established with extreme accuracy and this is why a standard bar is often used with two reflecting targets on the ends, which are oriented in several directions and are placed at varying distances from the laser tracker. This programme comprises 105 distance measurements which may be achieved in two different ways.

Both methods, however, share 41 of the 105 total points; they are the distance measurements and are evaluated using the Invar length reference bar, also known as a standard bar. The standard bar is moved at different orientations within a single plane (e.g., Plane $0, y, z$ ) and at various distances from the laser tracker while maintaining the angular encoders fixed at the origin $\left(0^{\circ}, 0^{\circ}, 0^{\circ}\right)$ in order to verify the distance, $d$ (as shown below) or $x$.
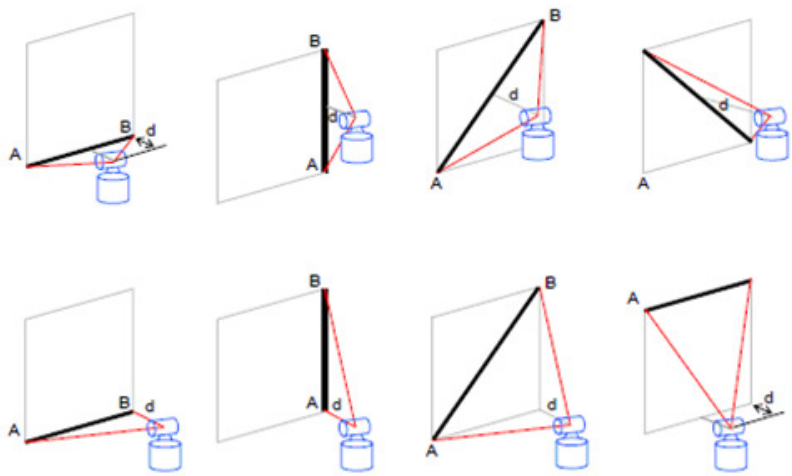

Figure 7.

Diagram of the 41 distance measurements common to both methods
For the remaining 64 measurement points, the two methods diverge in their approaches:

First method: Measuring the distance from the standard bar according to different orientations of the above.

Second method: 64 measurements made in a volume of $10 \mathrm{~m} \times 6 \mathrm{~m} \times 3 \mathrm{~m}$ (length $\mathrm{x}$ width $\mathrm{x}$ height) using eight measurement lines in space which are modelled by targets whose positions are known with perfect accuracy (see Figure 8 below).

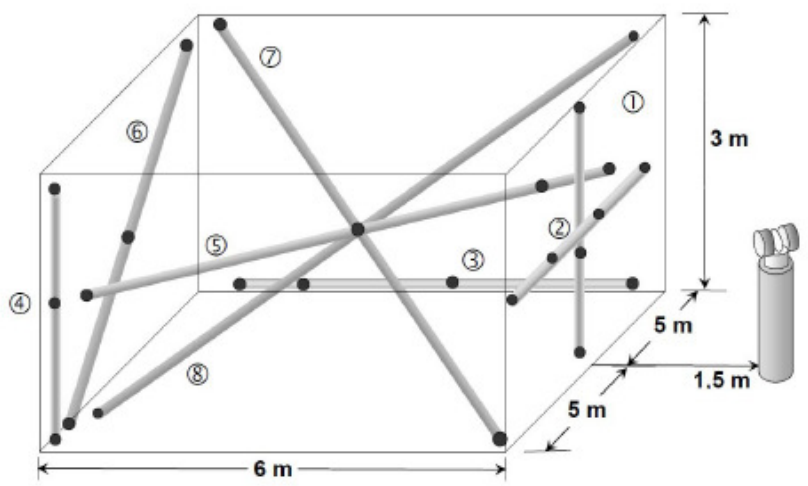

Figure 8. Diagram of the possible location of 8 measurement lines in a volume of $10 \mathrm{~m} \times 6 \mathrm{~m} \times 3 \mathrm{~m}$

This second method may also be achieved by fixing targets to a wall (see Figure 9 below).

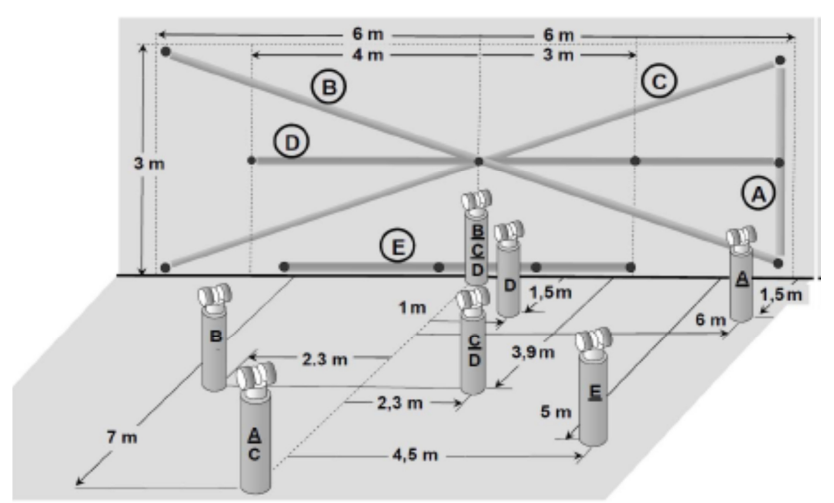

Figure 9. Diagram of the possible location of targets on a wall

$\begin{array}{cccc}\begin{array}{c}\text { Measuring } \\ \text { Line }\end{array} & \begin{array}{c}\text { Shortest } \\ \text { Test } \\ \text { Length (m) }\end{array} & \begin{array}{c}\text { Longest Test } \\ \text { Length (m) }\end{array} & \begin{array}{c}\text { Recommended } \\ \text { Longest Test } \\ \text { Length (m) }\end{array} \\ \text { A } & \geq 0.3 & 2.0 \leq l \leq 3.0 & 3.0 \\ \text { B } & \geq 0.3 & 8.1 \leq l \leq 12.4 & 12.0 \\ \text { C } & \geq 0.3 & 8.1 \leq l \leq 12.4 & 12.0 \\ \text { D } & \geq 0.3 & 6.6 \leq l \leq 10.0 & 9.0 \\ \text { E } & \geq 0.3 & 4.0 \leq l \leq 6.0 & 6.0\end{array}$

Table 3. Table giving details of the measurement lines required in the Fig. 8 above

When the 105 measurements of distance, $D_{\text {meas }}$, have been taken, subtract them from the reference distance, $D_{\text {ref, }}$ to obtain the length measurement error:

$$
E_{L}=D_{\text {meas }}-D_{\text {ref }}
$$


The maximum deviation between the measured distance and the reference distance is the length measurement error.

\subsection{Establishing conformity}

The standard states the conditions for establishing the metrological conformity of the equipment.

Probe size verification: All probe size errors $\mathrm{P}_{\mathrm{Si}}$ are less than the maximum permitted error for the size error $P_{S}$ MPE and all probe shape errors are less than the maximum permitted shape (form) error $\mathrm{P}_{\mathrm{F} \mathrm{MPE}}$.

$$
\mathrm{P}_{\mathrm{Si}} \leq \mathrm{P}_{\mathrm{S} \text { MPE }}
$$

However, if a single measurement indicates an error greater than the maximum permitted error, that measurement must be repeated three times and the greatest error must be less than the maximum permitted error.

Probe location verification: Probe location errors by the laser tracker $\mathrm{P}_{\mathrm{Li}}$ are less than the maximum permitted error for location error $\mathrm{P}_{\mathrm{L} \text { MPE }}$.

$$
\mathrm{P}_{\mathrm{Li}} \leq \mathrm{PL}_{\mathrm{MPE}}
$$

A double centering location error is acceptable. This location error must be re-measured three times and the greatest location error of these three must be less than the maximum permitted error.

Length measurements: Length measurement errors $\mathrm{E}_{\mathrm{Li}}$ should be less than the maximum permitted error on the length measurement error $\mathrm{E}_{\mathrm{L} \mathrm{MPE}}$.

$$
\mathrm{E}_{\mathrm{Li}} \leq \mathrm{E}_{\mathrm{LMPE}}
$$

No more than 5 of the 105 test lengths may be outside of specification. For any such length, 3 repeated measurements of any failed length must each be within the specification.

\subsection{Disadvantages of these standards}

\subsubsection{Standard ASME B89.4.19}

ASME B89.4.19 describes laser tracker verification programmes that focus on separating the three measurement parameters (azimuth encoder, zenithal encoder and distance meter) into independent systems, however they are truly co-dependent for threedimensional measurements.

\subsubsection{Draft standard ISO 10360-10}

This draft document has two significant disadvantages. First, the probe/reflector/target/SMR size verification method, which is identical to that for conventional $3 \mathrm{D}$ machine probes (MMT, Machine à mesurer tridimensionnelle) is not suitable for a $38.1 \mathrm{~mm}$ laser tracker probe. In fact, measuring 25 equally space points on a standard sphere with a diameter of between 30 and $50 \mathrm{~mm}$ is not at all an easy task. Second, implementation of the verification programme is very time-consuming. The laser tracker verification time is often competing with the down-time restrictions imposed by clients.

\section{Methods (manufacturers)}

This section deals with the verification and calibration protocols for the most common laser trackers used by the major manufacturers, Leica, Faro and API.

\subsection{Leica procedure}

Leica offers two types of software, Tracker Pilot ${ }^{\mathrm{TM}}$ and emScon $^{\mathrm{TM}}$ for verifying and calibrating their LT models. Tracker Pilot ${ }^{\mathrm{TM}}$ is used for the AT 401 model while emScon $^{\mathrm{TM}}$ is used for all other LT models (LTD 800, LTD 840 and AT 901).

\subsection{1 "Calibration" protocol LTD 800, LTD 840 and AT 901}

The verification and calibration of these laser tracker models is a long, painstaking process.

Using the emScon ${ }^{\mathrm{TM}}$ system software requires, in the first steps, the individual to carry out internal verification programmes with the software. If the results are noncompliant, adjustments have to be made using the appropriate self-calibration programmes, and the internal programmes have to be rerun to ensure that the corrections have, indeed, been made. Otherwise, one will have to run through the self-calibration programme, again. 


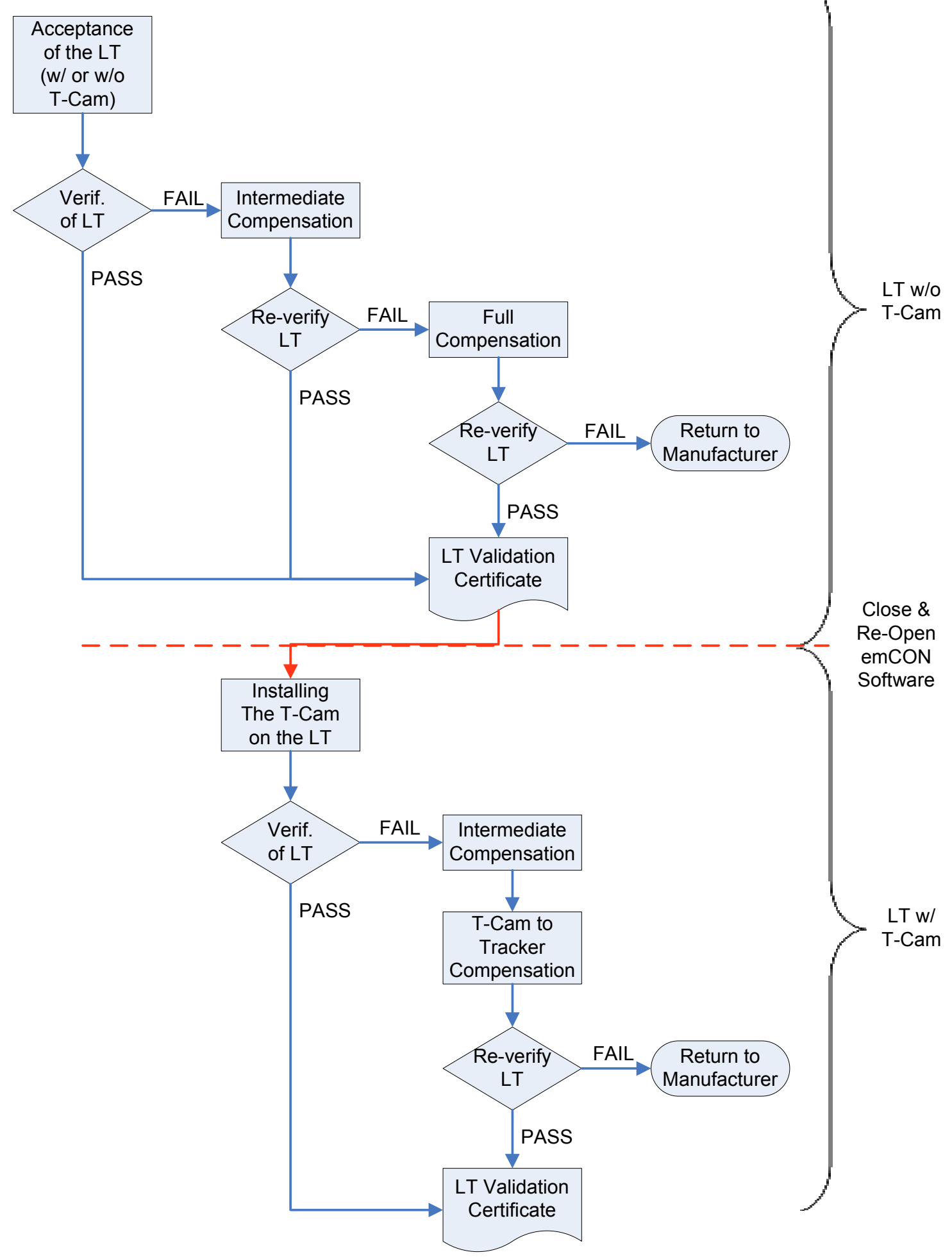

Figure 10. Diagram of the verification/calibration protocol for models LTD 800-840 and AT 901 


\subsubsection{AT 401 "calibration" protocol}

Calibrating the model AT 401 is quick and relatively easy with the Tracker Pilot ${ }^{\mathrm{TM}}$ operating software. This is explained by the fact that the AT 401 is a laser tracker that only uses ADM.

Initially, two verification programmes are run. If they are satisfactory, the laser tracker is compliant; otherwise an adjustment must be made.

On completion of the adjustment, the verification programmes are run again. If the laser tracker is found to be non-compliant, it is returned to the manufacturer for repair.

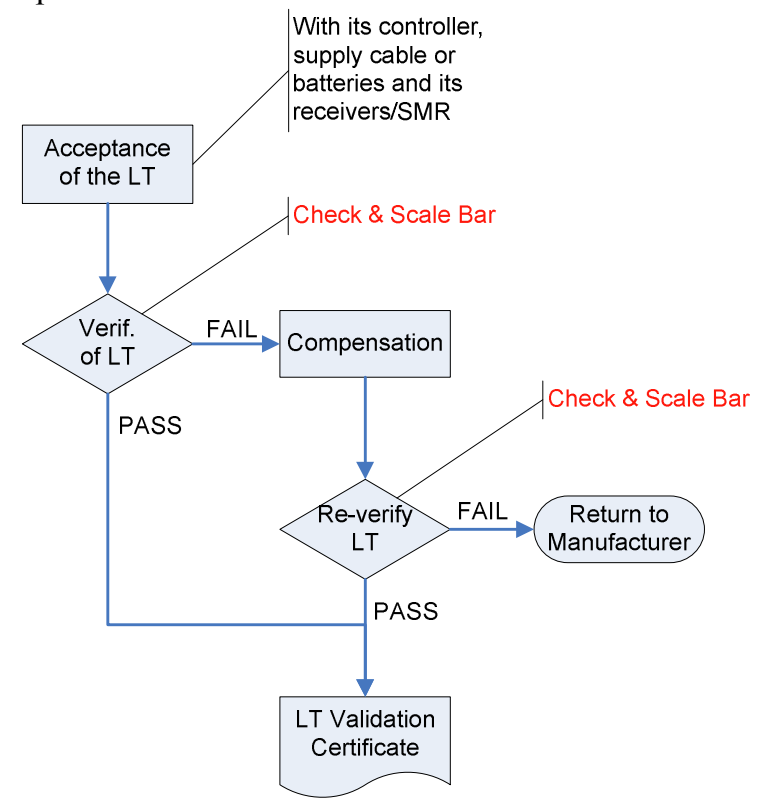

Figure 11. Diagram of the verification/ calibration protocol for model AT 401

\subsection{Faro procedure}

Unlike the Leica "calibration" procedure, the Faro protocol requires a prior self-calibration using its CompIT ${ }^{\mathrm{TM}}$ software.

Faro's "calibration" is also quick and simple, since the manufacturer only equips its models with the ADM (Absolute Distance Measurement) system (see section 2).

Calibration programmes are directly accessible by the operating software via "start-up checks" and "health checks".

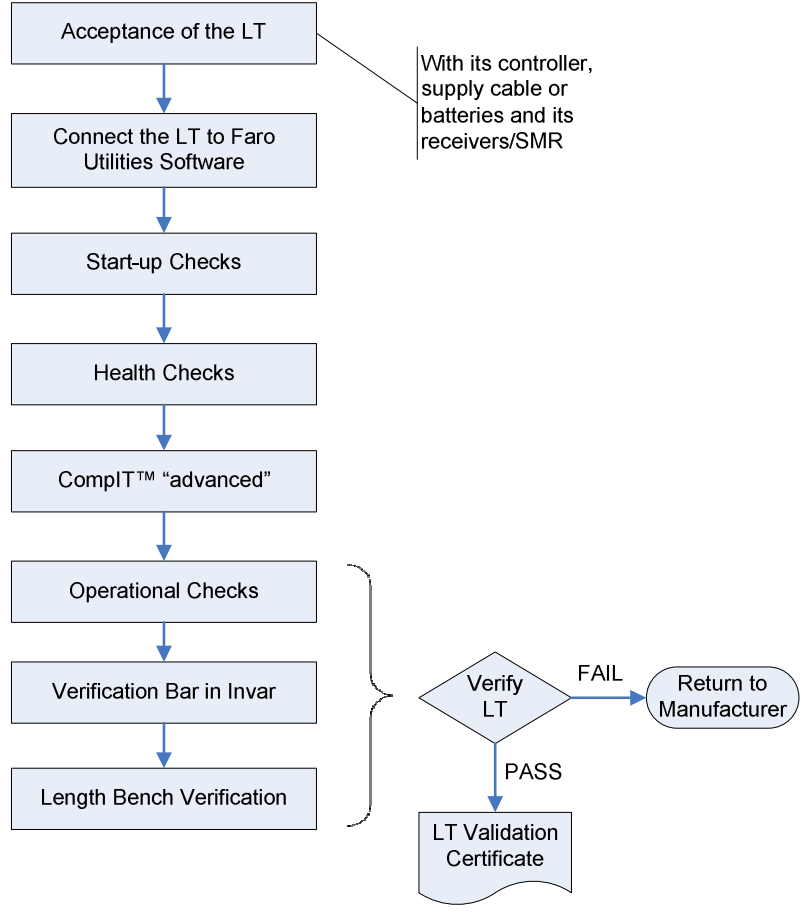

Figure 12. Diagram of the verification/ calibration protocol for the Faro Ion model

\subsection{API procedure}

API's laser trackers are equipped with both $\mathrm{ADM}$ and IFM.

Like Faro's "calibration" and verification procedure, API's is also quick and easy.

The calibration programs are directly accessible via the TrackerCal ${ }^{\mathrm{TM}}$ system software.

- $\mathrm{ADM}$ is verified at a minimum of five to ten points for long distance spreads over the distance to be measured, in comparison to the laser tracker's IFM.

- Angular verifications of the laser are done using the software's Backsight Check ${ }^{\mathrm{TM}}$ software. The procedure consists of pointing at a target/SMR at a distance of more than $2 \mathrm{~m}$ away. The tracker checks the aiming difference by double centring $\left(180^{\circ}\right)$ on each axis of rotation. The difference measured must be less than 0.0015 degrees

- If the angular difference is greater than $0.0015^{\circ}$ the manufacturer suggests carrying out a QVC (Quick Volumetric Calibration) at four points or if there is insufficient space then carrying out a simplified verification on a single point $(2 \mathrm{~m} \leq d$ $\leq 5 \mathrm{~m}$ from the laser head).

- The calibration file (PRM file) is updated in the controller with traceability of the operations carried out. 


\subsection{Disadvantages of the (manufacturers') methods}

- These methods do not comply with the basic calibration objective which is to identify, at a single point in time, the equipment's conformity in relation to the specifications, so as to detect any anomalies and to be able to deal with the effects on prior use of the device. Actually, the manufacturers suggest making adjustments (selfcalibration) prior to running the sequences. This causes reporting problems when "As Found" readings are critical and required by ISO 17025 , especially if the tracker was out of tolerance before the calibration (pre-alignment) sequence.

- The contents of the verification programme are limited to "self-calibration", measuring points by centring, measuring the length of calibration bars, etc. The traceability of these measurements in the International System of Units $(S I)$ could be better.

\section{Procedure / Trescal method}

To remedy the disadvantages of the methods described above, Trescal has set up methods based on the following steps:

- Complete calibration prior to any adjustment or self-check operations which includes verifying distances:

from a standard bar in a predetermined volume

with a long length in one axis.

The aim is to identify any anomalies and quantify measurement differences before making any adjustments.

- Verification and calibration in accordance with the manufacturers' protocols described in the previous section

- Again, full calibration as explained in the following sections.

\subsection{The methods used}

Trescal Vendôme in France (Central region) has a dimension laboratory with an area of $125 \mathrm{sq}$. m. and airconditioned to $20^{\circ} \mathrm{C} \pm 1{ }^{\circ} \mathrm{C}$. In addition to standards of lengths specific to this activity, it is equipped with a laser interferometer coupled to a $21 \mathrm{~m}$ length bench. This bench allows accredited calibration and verification operations to be performed with a laser distance meter.

\subsection{Calibration by measuring a standard bar}

This calibration takes inspiration from draft standard ISO 10360-10 on the length measurement section (see 3.1.2.1.3). However, for time saving and efficiency reasons, only measurements made as close as possible to the laser tracker $(\sim 1.5 \mathrm{~m})$ are used (items $1,2,8-11$ and $12-15$ of the standard). These verifications correspond to the measurement of a standard bar of approximately 2.05 $\mathrm{m}$ in the horizontal, vertical and each diagonal position. It allows angular encoders to be tested at the maximum of the measuring capability. (Critical measurements)

The measurement uncertainty for the distances measured is $0.019 \mathrm{~mm}$.
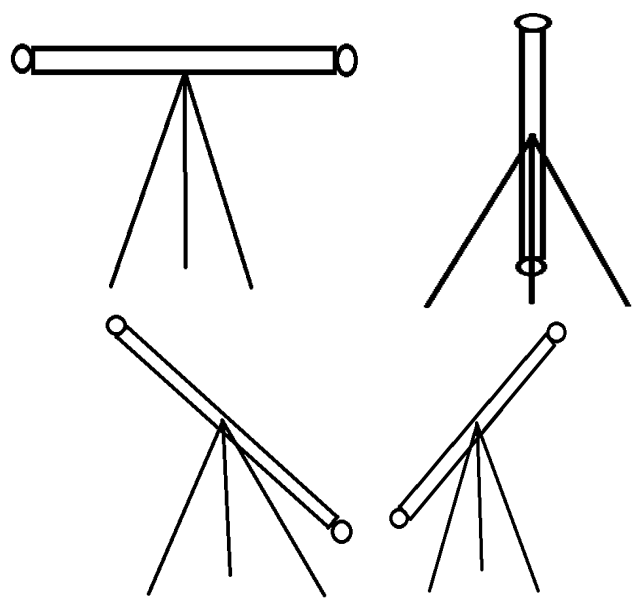

Figure 13. Diagram of the orientation of the standard bar for distance measurements

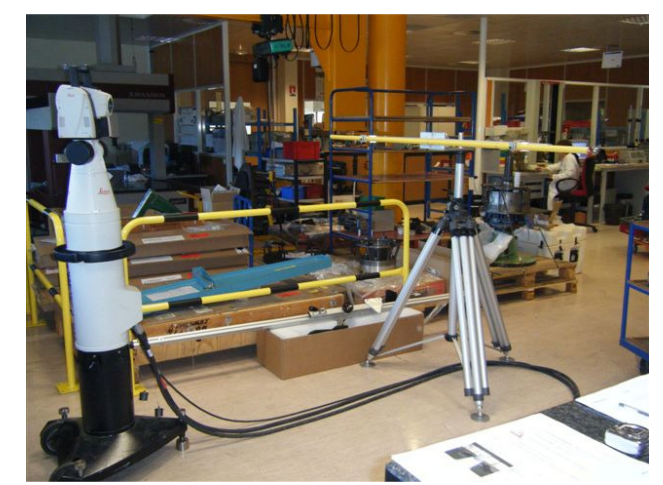

Figure 14. Distance measurement of a standard bar

\subsection{Calibration by comparison with a laser interferometer}

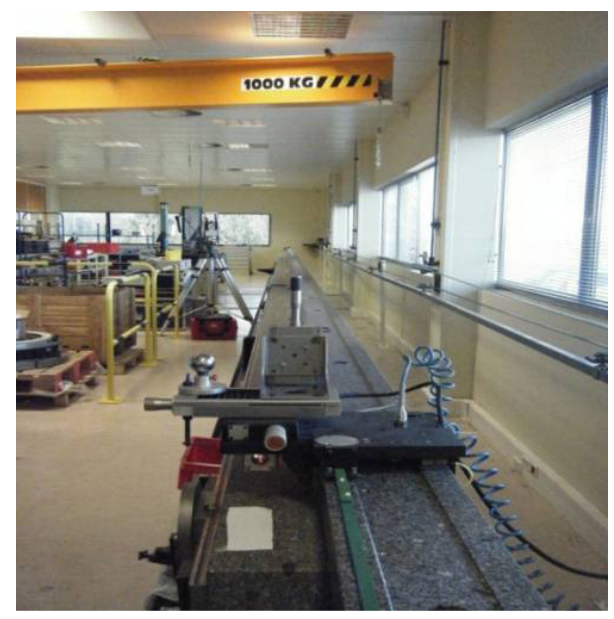

Figure 15. View of $21 \mathrm{~m}$ bench 
The calibration by comparison with a laser interferometer consists of placing the laser tracker alongside the laser interferometer and placing the reflector/target/SMR on the mobile carriage and then comparing the distance measurements given by the LT to the standard distances given by the laser interferometer.

This metrological confirmation meets linear distance verifications in the draft ISO 10360-10 standard (positions 36-40).

The measurement uncertainty for the distances measured is: $0.02 \mathrm{~mm}+10.10^{-6} * L$, where $L$ is the measured distance.

\subsection{Advantage of the Trescal method}

Given the economic constraints, our experience has led us to limit verification programmes and the number of measurement points to only what is necessary. Also, these can be adapted to the customer's needs.

In summary :

$>$ To meet the needs of our customers, in a "Fast paced society", Trescal

$>$ Maintains an auditable measurement programme,

$>$ Implements appropriate standards and methods,

$>$ Adjusts the number of measurement points, and

$>$ Reduces equipment downtime and cost of metrological services.

\section{Conclusion}

To meet its own needs and those of its customers, the laser tracker calibration method used by Trescal is based on an approach which allows the expertise resulting from standardisation to be analysed and taken into consideration, as well as product designers and actual customer needs which are minimizing down-time and calibration service costs. 